\title{
PROBABILITY ESTIMATION DEGREE OF DANGER VESSELS RAPPROCHEMENT
}

\section{ОЦЕНКА ВЕРОЯТНОСТИ СТЕПЕНИ ОПАСНОСТИ СБЛИЖЕНИЯ СУДОВ}

\author{
I. A. Burmaka, PhD, associate professor, A.V. Yanchetskyy, PhD student \\ И.А. Бурмака, к.т.н., дочент, А.В. Янчецкий, аспирант \\ National University "Odessa Maritime Academy», Ukraine \\ Начиональный университет «Одесская морская академия», Украина
}

\begin{abstract}
In the article is specified, that a decision-making process contains the following stages: control of environment, including relative position and parameters of relative motion, the exposure of situation of rapprochement of vessels, in the case of rapprochement of vessels it is necessary to produce estimation of degree of his danger, at dangerous rapprochement is needed to define the type of co-operation, depending on the degree of danger of situation of rapprochement the choice of strategy of divergence is produced.

On the first stage of process of decision-making surrounding mobile objects come to light by ARPA or AIS, for which is measured parameters of motion and relative position. It is shown that on the second stage of process of decision-making, using the measured parameters, it is necessary to expect the value of speed of change of distance between vessels, taking into account that at its negative value ships are drawn together. On the third stage of process of decision-making at rapprochement of vessels estimation of degree of his danger is produced, what development of situation of rapprochement to the moment of time of their shortest rapprochement is forecast for.

On the fourth stage of decision-making it is necessary to define the type of cooperation of the drawn together vessels, taking into account principle their process control of divergence. It is especially substantial for principle of locally-independent management by the process of divergence, when coordination of co-operation of vessels at dangerous rapprochement is needed. The fifth stage of decision-making is characterized by the choice of strategy of divergence, thus at the locally-independent process control of divergence the choice of strategy of divergence is produced depending on the degree of danger of situation of rapprochement, by the standard maneuver of divergence or for excessive rapprochement of vessels it is necessary to use the maneuver of urgent divergence.

In work as the index of efficiency of the analytical collision avoidance systems vessels probability of safe completion of process of divergence, which is work of probabilities of successful end of stages of process of decision-making on the choice of strategy of divergence, is offered.

It is shown that first three stages of decision-making on the choice of strategy of divergence are characterized by general probability of absence of danger of collision on condition that distance of the shortest rapprochement is equal to the set minimum-possible distance of rapprochement. For determination of the mentioned probability the error of distance of the shortest rapprochement is considered and expression is got for the closeness of its distributing. To that end collected dependence of error of distance of the shortest rapprochement from the errors of measuring of distance and bearing.

By the got expression for the closeness of distributing of error of distance of the shortest rapprochement probability is certain of that at equality of distance of the shortest rapprochement with limit-possible distance of rapprochement there will not be the collision.
\end{abstract}

Keywords: safety of navigation, warning of collision of vessels, index of efficiency of the analytical collision avoidance systems vessels, probability of absence of danger of collision. 


\section{PЕФЕРАТ}

В статті вказується, що процес ухвалення рішення містить наступні етапи: контроль навколишнього оточення, включаючи відносну позицію і параметри відносного руху, виявлення ситуації зближення суден, у разі зближення суден слід провести оцінку ступеня його небезпеки, при небезпечному зближенні необхідне визначити тип взаємодії, залежно від ступеня небезпеки ситуації зближення здійснити вибір стратегії розходження.

На першому етапі процесу ухвалення рішення за допомогою ЗАРП або АIC виявляються навколишні рухомі об'єкти, для яких вимірюються параметри руху і відносного положення. Показано, що на другому етапі процесу ухвалення рішення, використовуючи зміряні параметри, необхідно розрахувати значення швидкості зміни дистанції між суднами, враховуючи, що при ії негативному значенні судна зближуються. На третьому етапі процесу ухвалення рішення при зближенні суден проводиться оцінка ступеня його небезпеки, для чого прогнозується розвиток ситуації зближення до моменту часу їх найкоротшого зближення.

На четвертому етапі ухвалення рішення слід визначити тип взаємодії суден, що зближуються, враховуючи принцип управлінні їх процесом розходження. Особливо істотно це для принципу локально-незалежного управління процесом розходження, коли необхідна координація взаємодії суден при небезпечному зближенні. П'ятий етап ухвалення рішення характеризується вибором стратегії розходження, причому при локально-незалежному управлінні процесом розходження вибір стратегії розходження проводиться залежно від ступеня небезпеки ситуації зближення, тобто стандартним маневром розходження або при надмірному зближенні суден слід використовувати маневр екстреного розходження.

У роботі як показник ефективності аналітичних систем попередження зіткнень суден запропонована вірогідність безпечного завершення процесу розходження, яка $є$ добутком вірогідності успішного результату етапів процесу ухвалення рішення по вибору стратегії розходження.

Показано, що перші три етапи ухвалення рішення по вибору стратегії розходження характеризуються загальною вірогідністю відсутності небезпеки зіткнення за умови, що дистанція найкоротшого зближення рівна заданій гранично - допустимій дистанції зближення. Для визначення згаданої вірогідності розглянута похибка дистанції найкоротшого зближення і одержано вираз для щільності їі розподілу. 3 цією метою одержана залежність похибки дистанції найкоротшого зближення від похибок вимірювання дистанції і пеленга.

За допомогою одержаного виразу для щільності розподілу похибки дистанції найкоротшого зближення визначена вірогідність того, що при рівності дистанції найкоротшого зближення 3 гранично - допустимою дистанцією зближення не виникне зіткнення.

Ключові слова: безпека судноводіння, попередження зіткнення суден, показник ефективності аналітичних систем попередження зіткнень суден, вірогідність відсутності небезпеки зіткнення.

\section{Постановка проблемы в общем виде и ее связь с важными научными или практическими задачами}

В случае опасного сближения судов требуется оценить ситуацию сближения и оперативно выбрать безопасный маневр расхождения, который соответствовал бы хорошей морской практике. Благодаря научно-техническому прогрессу в настоящее время предложены различные типы аналитических систем предупреждения столкновений судов, что обуславливает необходимость разработки способа сравнительного анализа их эффективности. Одним из существенных вопросов такой разработки является оценка вероятности степени опасности сближении судов, чем обоснован выбор темы статьи. 
Анализ последних достижений и публикаций, в которых начато решение данной проблемы и выделение нерешенных ранее частей общей проблемы

Принципы локально-независимого и полного внешнего управления процессом расхождения опасно сближающихся судов рассмотрены в работе [1], а в работе [2] предложен выбор ситуации сближения судна с целью из множества стандартных ситуаций, после чего производится определение стратегии расхождения. Подробное исследование методов локально-независимого управления приведено в работе [3], причем для расхождения оперирующего судна с несколькими опасными целями предложен метод формирования гибких стратегий расхождения. В работе [4] рассмотрены взаимодействие судов при опасном сближении и выбор стратегии расхождения для предупреждения их столкновения.

Работа [5] посвящена автономной судовой системе уклонения от столкновения, в которой рассматриваются требования к автономной навигации и указывается, что исследования по автоматизации управления судном могут быть представлены в классической или компьютерной категориях.

Вопросам учета навигационных опасностей и инерционности судна при выборе стратегии расхождения судна посвящены работы [6,7], а в работе [8] предложено описание процесса расхождения судов в терминах дифференциальной антагонистической игры.

В монографии [9] приведен метод предупреждения столкновения судов путем смещения на параллельную линию пути, а экстренная стратегия расхождения в ситуации чрезмерного сближении судов предложена в работе [10].

Результаты исследования эффективности парных маневров расхождения приведены в работе [11], а в работе [12] рассмотрено управления тремя суднами для безопасного расхождения.

\section{Формулирование целей статьи (постановка задачи)}

Целью статьи является рассмотрение процесса принятия решения о выборе стратегии расхождения и оценке вероятности степени опасности сближения судов.

\section{Изложение материала исследования с обоснованием полученных научных результатов}

Процесс принятия решения о выборе стратегии расхождения содержит следующие этапы:

- контроль окружающей обстановки, включая относительную позицию и параметры относительного движения;

- выявление ситуации сближения судов;

- при сближении судов следует произвести оценку степени его опасности;

- в случае опасного сближения определить тип взаимодействия;

- в зависимости от степени опасности ситуации сближения выбор стратегии расхождения.

На первом этапе процесса принятия решения с помощью САРП или АИС выявляются окружающие подвижные объекты, для которых измеряются параметров движения и относительного положения. На этом этапе определяются относительный курс $\mathrm{K}_{\mathrm{ot}}$ и скорость $\mathrm{V}_{\mathrm{ot}}$ с помощью выражений:

$$
\begin{gathered}
\mathrm{K}_{\mathrm{ot}}=\arcsin \left[\left(\mathrm{V}_{1} \sin \mathrm{K}_{1}-\mathrm{V}_{2} \sin \mathrm{K}_{2}\right) / \mathrm{V}_{\mathrm{ot}}\right], \\
\mathrm{V}_{\mathrm{ot}}=\left[\mathrm{V}_{1}^{2}+\mathrm{V}_{2}^{2}-2 \mathrm{~V}_{1} \mathrm{~V}_{2} \cos \left(\mathrm{K}_{1}-\mathrm{K}_{2}\right)\right]^{1 / 2},
\end{gathered}
$$

где $\mathrm{K}_{1}, \mathrm{~V}_{1}, \mathrm{~K}_{2}$ и $\mathrm{V}_{2}$ - параметры движения судов.

На втором этапе процесса принятия решения, используя измеренные параметры, необходимо рассчитать значение скорости изменения дистанции между суднами Dं, 
учитывая, что при ее отрицательном значении суда сближаются. Значение скорости изменения дистанции между суднами рассчитывается с помощью выражения:

$$
\dot{\mathrm{D}}=-\mathrm{V}_{\mathrm{ot}} \cos \left(\alpha-\mathrm{K}_{\mathrm{ot}}\right)
$$

где $\alpha$ - пеленг с одного судна на другое судно.

На третьем этапе процесса принятия решения при сближении судов производится оценка степени его опасности, для чего прогнозируется развитие ситуации сближения до момента времени их кратчайшего сближения, которое характеризуется дистанцией $\mathrm{D}_{\min }$ :

$$
\mathrm{D}_{\text {min }}=\left|\mathrm{D} \sin \left(\alpha-\mathrm{K}_{\mathrm{ot}}\right)\right|
$$

где D - дистанция между суднами.

Степень опасности сближении судов характеризуется ситуационным возмущением $\omega$, которое возникает при прогнозируемом попадании судов в домен недопустимых позиций. Оно возникает тогда, когда прогнозируемое значение дистанции кратчайшего сближения $\mathrm{D}_{\text {min }}$ меньше значения предельно-допустимой дистанции сближения $\mathrm{D}_{\mathrm{d}}$, величина которой зависит от формы домена недопустимых позиций и ракурса сближения судов. При опасном сближении ситуационное возмущение $\omega$ может принимать значения 1 или 2.

После того, как выявлено наличие опасности столкновения, на четвертом этапе принятия решения следует определить тип взаимодействия сближающихся судов, учитывая принцип управлении их процессом расхождения. Особенно существенно это для принципа локально-независимого управления процессом расхождения, когда необходима координация взаимодействия судов при опасном сближении.

На пятом этапе принятия решения следует выбрать стратегию расхождения, причем при локально-независимом управлении процессом расхождения выбор стратегии расхождения производится в зависимости от степени опасности ситуации сближения, т. е. значения ситуационного возмущения $\omega>0$, исходя из координации маневров сближающихся судов, предусмотренной МППСС-72. В этом случае характер маневра расхождения также определяется значением ситуационного возмущения. В случае $\omega=1$ предусмотрено применение стандартного маневра расхождения, а при значении $\omega=2$, что характерно для чрезмерного сближения судов, во избежание столкновения следует использовать маневр экстренного расхождения.

Принципиально важным является число опасно сближающихся судов. В случае, когда опасно сближаются более двух судов, стратегия расхождения формируется с учетом матрицы ситуационного возмущения, которая в качестве элемента содержит значение ситуационного возмущения соответствующей пары судов.

В качестве показателя эффективности аналитических систем предупреждения столкновений судов целесообразно предложить вероятность безопасного завершения процесса расхождения $\mathrm{P}_{\mathrm{sf}}$, которая является произведением вероятностей $\mathrm{P}_{\mathrm{i}}$ успешного исхода этапов процесса принятия решения по выбору стратегии расхождения:

$$
\mathrm{P}_{\mathrm{sf}}=\mathrm{P}_{1} \mathrm{P}_{2} \mathrm{P}_{3} \mathrm{P}_{4} \mathrm{P}_{5}
$$

Отметим, что первые три этапа принятия решения по выбору стратегии расхождения можно характеризовать общей вероятностью отсутствия опасности столкновения при условии, что дистанция кратчайшего сближения равна заданной предельно-допустимой 
дистанции сближения. Для определения упомянутой вероятности следует рассмотреть погрешность дистанции кратчайшего сближения $\Delta \mathrm{D}_{\min }$ и найти выражение для плотности ее распределения.

С этой целью следует рассмотреть зависимость погрешности дистанции кратчайшего сближения $\Delta \mathrm{D}_{\min }$ от погрешностей измерения дистанции $\Delta \mathrm{D}$ и пеленга $\Delta \alpha$. Для этого воспользуемся выражением для $\mathrm{D}_{\min }$ :

$$
\mathrm{D}_{\text {min }}=\left|\mathrm{D} \sin \left(\alpha-\mathrm{K}_{\mathrm{ot}}\right)\right|
$$

и с учетом соответствующих погрешностей $\Delta \mathrm{D}_{\min }, \Delta \mathrm{D}$ и $\Delta \alpha$ получим:

$$
\begin{gathered}
\mathrm{D}_{\text {min }}+\Delta \mathrm{D}_{\text {min }}=\left|(\mathrm{D}+\Delta \mathrm{D}) \sin \left[\left(\alpha-\mathrm{K}_{\mathrm{ot}}\right)+\Delta \alpha\right]\right|=(\mathrm{D}+\Delta \mathrm{D})\left[\sin \left(\alpha-\mathrm{K}_{\mathrm{ot}}\right) \cos \Delta \alpha+\right. \\
\left.+\cos \left(\alpha-\mathrm{K}_{\mathrm{ot}}\right) \sin \Delta \alpha\right] .
\end{gathered}
$$

Так как погрешности измерения имеют малые значения, то можно принять $\cos \Delta \alpha=1$ и $\sin \Delta \alpha=\Delta \alpha$. Следовательно,

$$
\begin{gathered}
\mathrm{D}_{\text {min }}+\Delta \mathrm{D}_{\text {min }}=(\mathrm{D}+\Delta \mathrm{D})\left[\sin \left(\alpha-\mathrm{K}_{\mathrm{ot}}\right)+\cos \left(\alpha-\mathrm{K}_{\mathrm{ot}}\right) \Delta \alpha\right]=\mathrm{D}\left|\sin \left(\alpha-\mathrm{K}_{\mathrm{ot}}\right)\right|+ \\
\mathrm{D} \cos \left(\alpha-\mathrm{K}_{\mathrm{ot}}\right) \Delta \alpha+\Delta \mathrm{D}\left|\sin \left(\alpha-\mathrm{K}_{\mathrm{ot}}\right)\right|+\Delta \mathrm{D} \cos \left(\alpha-\mathrm{K}_{\mathrm{ot}}\right) \Delta \alpha .
\end{gathered}
$$

Обращаем внимание, что в последнем выражении:

$$
\mathrm{D}\left|\sin \left(\alpha-\mathrm{K}_{\mathrm{ot}}\right)\right|=\mathrm{D}_{\min }, \Delta \mathrm{D} \cos \left(\alpha-\mathrm{K}_{\mathrm{ot}}\right) \Delta \alpha \approx 0
$$

учитывая, что $\Delta \mathrm{D}$ и $\Delta \alpha$ являются величинами с малыми значениями. Поэтому:

$$
\mathrm{D}_{\text {min }}+\Delta \mathrm{D}_{\text {min }}=\mathrm{D}_{\text {min }}+\mathrm{D} \cos \left(\alpha-\mathrm{K}_{\mathrm{ot}}\right) \Delta \alpha+\Delta \mathrm{D}\left|\sin \left(\alpha-\mathrm{K}_{\mathrm{ot}}\right)\right| \text {, }
$$

или окончательно:

$$
\Delta \mathrm{D}_{\min }=\mathrm{D} \cos \left(\alpha-\mathrm{K}_{\mathrm{ot}}\right) \Delta \alpha+\Delta \mathrm{D}\left|\sin \left(\alpha-\mathrm{K}_{\mathrm{ot}}\right)\right|
$$

Такой же результат получим альтернативным путем. Найдем полный дифференциал выражения (1):

$$
\mathrm{d}\left(\mathrm{D}_{\text {min }}\right)=\left|\mathrm{d}(\mathrm{D}) \sin \left(\alpha-\mathrm{K}_{\mathrm{ot}}\right)\right|+\operatorname{Dcos}\left(\alpha-\mathrm{K}_{\mathrm{ot}}\right) \mathrm{d} \alpha
$$

Переходя от дифференциалов к конечным приращениям, получим выражение (2).

С учетом зависимости (2) и предполагая, что погрешности $\Delta \mathrm{D}$ и $\Delta \alpha$ распределены по нормальному закону с дисперсиями $\sigma_{\mathrm{D}}^{2}$ и $\sigma_{\alpha}^{2}$ можно записать выражение для дисперсии погрешности $\Delta \mathrm{D}_{\min }$, которую обозначим $\sigma_{\min }^{2}$. Поэтому:

$$
\sigma_{\min }^{2}=\sigma_{\mathrm{D}^{2}}^{2} \sin ^{2}\left(\alpha-\mathrm{K}_{\mathrm{ot}}\right)+\sigma_{\alpha}^{2} \mathrm{D}^{2} \cos ^{2}\left(\alpha-\mathrm{K}_{\mathrm{ot}}\right) \text {. }
$$

В этом случае закон распределения погрешности $\Delta \mathrm{D}_{\min }$ также является нормальным и плотность $\mathrm{f}_{\mathrm{b}}\left(\Delta \mathrm{D}_{\min }\right)$ выражается следующим образом: 


$$
\mathrm{f}_{\mathrm{b}}\left(\Delta \mathrm{D}_{\min }\right)=\frac{1}{\sqrt{2 \pi} \sigma_{\min }} \exp \left[-\frac{\left(\Delta \mathrm{D}_{\min }\right)^{2}}{2 \sigma_{\min }^{2}}\right]
$$

или

$$
\mathrm{f}_{\mathrm{b}}\left(\Delta \mathrm{D}_{\min }\right)=\mathrm{A}_{\mathrm{n}} \exp \left\{-\frac{\left[\Delta \mathrm{D}_{\min }\right]^{2}}{2\left[\sigma_{\mathrm{D}}^{2} \sin ^{2}\left(\alpha-\mathrm{K}_{\mathrm{ot}}\right)+\sigma_{\alpha}^{2} \mathrm{D}^{2} \cos ^{2}\left(\alpha-\mathrm{K}_{\mathrm{ot}}\right)\right)}\right\}
$$

где $\mathrm{A}_{\mathrm{n}}=\frac{1}{\sqrt{2 \pi\left(\sigma_{\mathrm{D}}^{2} \sin ^{2}\left(\alpha-\mathrm{K}_{\mathrm{ot}}\right)+\sigma_{\alpha}^{2} \mathrm{D}^{2} \cos ^{2}\left(\alpha-\mathrm{K}_{\mathrm{ot}}\right)\right)}}$.

Располагая выражением для плотности распределения $\mathrm{f}_{\mathrm{b}}\left(\Delta \mathrm{D}_{\mathrm{min}}\right)$, можно определить вероятность $\mathrm{P}_{\mathrm{b}}$ того, что в случае равенства $\mathrm{D}_{\min }=\mathrm{D}_{\mathrm{d}}$ при сближении судов не возникнет столкновения. Для этого обратимся к рис. 1, на котором обозначено через $\mathrm{D}_{\mathrm{dt}}$ радиус детерминированной области, учитывающей габариты судна и явление присасывания.

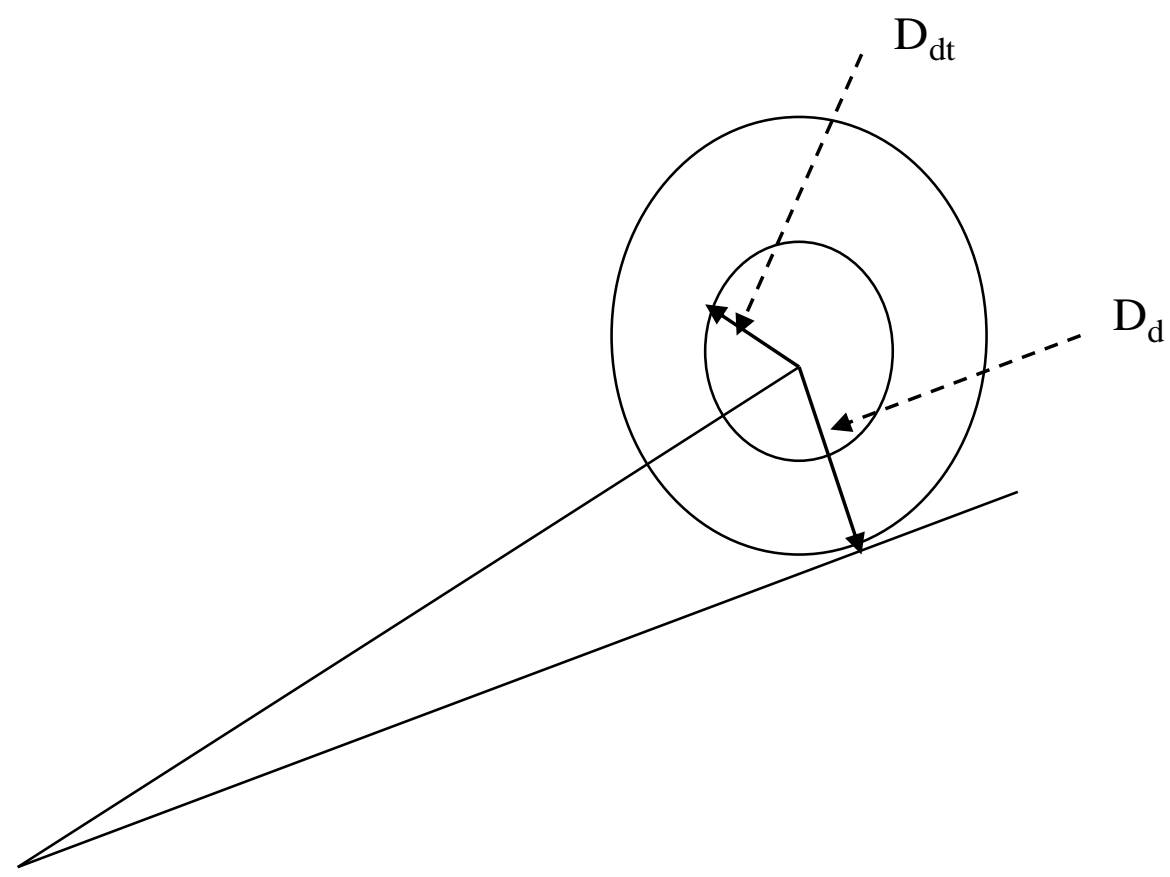

Рис. 1. Определение вероятности безаварийного сближения

Очевидно, искомая вероятность равна вероятности того, что погрешность $\Delta \mathrm{D}_{\min }$ не превзойдет величину $\mathrm{D}_{\min }-\mathrm{D}_{\mathrm{dt}}, \quad$ т. е. $\mathrm{P}_{\mathrm{b}}=\mathrm{P}\left\{\Delta \mathrm{D}_{\min }<\mathrm{D}_{\min }-\mathrm{D}_{\mathrm{dt}}\right\}$. C учетом полученного выражения (3) для плотности распределения $\mathrm{f}_{\mathrm{b}}\left(\Delta \mathrm{D}_{\min }\right)$ получим:

$$
\begin{gathered}
\mathrm{P}_{\mathrm{b}}=\mathrm{P}\left\{\Delta \mathrm{D}_{\text {min }}<\mathrm{D}_{\text {min }}-\mathrm{D}_{\mathrm{dt}}\right\}=\int_{0}^{\mathrm{D}_{\text {min }}-\mathrm{D}_{\mathrm{dt}}} \mathrm{f}_{\mathrm{b}}\left(\Delta \mathrm{D}_{\text {min }}\right) \mathrm{d} \Delta \mathrm{D}_{\text {min }}, \text { или } \\
\mathrm{P}_{\mathrm{b}}=\int_{0}^{\mathrm{D}_{\text {min }}-\mathrm{D}_{\mathrm{dt}}} \mathrm{f}_{\mathrm{b}}\left(\Delta \mathrm{D}_{\text {min }}\right) \mathrm{d} \Delta \mathrm{D}_{\text {min }} .
\end{gathered}
$$


В случае домена с формой, отличной от круга, необходимо рассматривать вопрос определения $\mathrm{D}_{\mathrm{dt}}$. Полученная вероятность $\mathrm{P}_{\mathrm{b}}$ определяет благоприятный исход первого этапа.

\section{Выводы и перспектива дальнейшей работы по данному направлению}

Таким образом, в данной статье рассмотрен процесс принятия решения о выборе стратегии расхождения и предложена процедура оценки вероятности степени опасности сближения судов. В дальнейшем целесообразно рассмотреть возможность определения вероятностей успешного исхода остальных этапов процесса принятия решения по выбору стратегии расхождения.

\section{ЛИТЕРАТУРА}

1. Бурмака И.А. Управление судами в ситуации опасного сближения / И.А Бурмака., Э.Н Пятаков, А.Ю. Булгаков - LAP LAMBERT Academic Publishing, - Саарбрюккен (Германия), - 2016. - 585 с.

2. Мальцев А. С. Маневрирование судов при расхождении / А.С. Мальцев, Е.Е. Тюпиков, И.И. Ворохобин - Одесса: Морской тренажерный центр, 2013. - 304 с.

3. Цымбал Н.Н. Гибкие стратегии расхождения судов / Н.Н. Цымбал, И.А. Бурмака, Е.Е. Тюпиков. - Одесса: КП ОГТ, 2007. - 424 с.

4. Пятаков Э.Н. Взаимодействие судов при расхождении для предупреждения столкновения / Пятаков Э.Н., Бужбецкий Р.Ю., Бурмака И.А., Булгаков А.Ю. - Херсон: Гринь Д.С., 2015. - 312 с.

5. Statheros Thomas. Autonomous ship collision avoidance navigation concepts, technologies and techniques / Statheros Thomas, Howells Gareth, McDonald-Maier Klaus. // J. Navig. 2008. 61, № 1, p. 129-142.

6. Петриченко Е.А. Вывод условия существования множества допустимых маневров расхождения с учетом навигационных опасностей / Петриченко Е.А. // Судовождение. - 2003. - №.6. - C. 103 - 107.

7. Бурмака И.А. Результаты имитационного моделирования процесса расхождения судов с учетом их динамики / Бурмака И.А. // Судовождение. - 2005. - №10. - С. 21 - 25.

8. Lisowski J. Game and computational intelligence decision making algorithms for avoiding collision at sea/ Lisowski J. // Proc. of the IEEE Int. Conf. on Technologies for Homeland Security and Safety. - 2005. - Gdańsk. - P. 71 - 78.

9. Вагущенко Л.Л. Расхождение с суднами смещением на параллельную линию пути / Л.Л. Вагущенко. - Одесса: Фенікс, 2013. - 180 с.

10. Бурмака И.А. Экстренная стратегия расхождения при чрезмерном сближении судов / Бурмака И.А., Бурмака А. И., Бужбецкий P.Ю. - LAP LAMBERT Academic Publishing, 2014. - 202 c.

11. Пятаков Э.Н. Оценка эффективности парных стратегий расходящихся судов / Э.Н. Пятаков., С.И. Заичко // Судовождение: Сб. научн. трудов. / ОНМА, - Вып.15. - Одесса: "ИздатИнформ", 2008. - С. 166 - 171.

12. Бурмака И.А. Маневр расхождения трех судов изменением курсов/ И.А. Бурмака, А.Ю. Булгаков // Автоматизация судовых технических средств: науч. -техн. сб. - 2014. - Вып. 20. Одесса: ОНМА. - С. 18 -23. 